\title{
Complete nucleotide sequence of the S10 genome segment of grass carp reovirus (GCRV)*
}

\author{
Tao Qiu, Ren-Hou Lu**, Jing Zhang, Zuo-Yan Zhu
}

State Key Laboratory of Freshwater Ecology and Biotechnology, Institute of Hydrobiology, Chinese Academy of Sciences, Wuhan 430072, China

\begin{abstract}
Hemorrhagic disease, caused by the grass carp reovirus (GCRV), is one of the major diseases of grass carp in China. Little is known about the structure and function of the gene segments of this reovirus. The S10 genome segment of GCRV was cloned and the complete nucleotide sequence is reported here. The S10 is 909 nucleotides long and contains a large open reading frame (ORF) encoding a protein of 276 amino acids with a deduced molecular weight of approximately $29.7 \mathrm{kDa}$. Comparisons of the deduced amino acid sequence of GCRV S10 with those of other reoviruses revealed no significant homologies. However, GCRV S10 shared a putative zinc-finger sequence and a similar distribution of hydrophilic motifs with the outer capsid proteins encoded by Coho salmon aquareovirus (SCSV) S10, striped bass reovirus (SBRV) S10, and mammalian reovirus (MRV) S4. It was predicted that this segment gene encodes an outer capsid protein.
\end{abstract}

KEY WORDS: Grass carp reovirus (GCRV) - S10 genome segment $\cdot$ Nucleotide sequence

Resale or republication not permitted without written consent of the publisher

Grass carp reovirus (GCRV), an important fish pathogen involved in hemorrhagic disease (Chen \& Jiang 1983), not only infects grass carp Ctenopharyngodon idellus, but also was found capable of infecting black carp Mylopharyngodon piceus, topmouth gudeon Pseudorasbora parva (Ding et al. 1991) and rare minnow Gobiocypris rarus (Wang et al. 1994), and historically has resulted in large losses in freshwater fish culture in China. The virions are resistant to chloroform and ether, non-sensitive to acid ( $\mathrm{pH} 3$ ) and alkaline ( $\mathrm{pH} 10)$ treatment, and stable within a certain range of temperature. The virus belongs to the genus Aquareo-

*The nucleotide sequence data for GCRV S10 reported in this paper has been deposited in the GenBank data base under the accession number AF236688.

${ }^{* *}$ Corresponding author. E-mail: rhlu@ihb.ac.cn virus, as a tentative member, and shares the physicochemical properties and morphological characteristics of the family Reoviridae. About 10 isolates reported to date (Li et al. 1999) had different electrophoretic patterns but the same number of genomic dsRNA. The GCRV possesses a double-stranded dsRNA genome consisting of 11 segments packaged into a nonenveloped icosahedral double capsid approximately 55 to $80 \mathrm{~nm}$ in diameter (Ke et al. 1990). The genome segments are approximately 25000 nucleotide pairs in total size deduced by the dsRNA molecular weight and separated into 3 size classes: large (L1, L2, and L3); medium (M4, M5, and M6); and small (S7, S8, S9, S10, and $\mathrm{S} 11$ ) in order of mobility in polyacrylamide gels from the slowest to fastest, respectively.

Many reoviruses capable of infecting aquatic organisms have been identified (Plumb et al. 1979, Hedrick et al. 1984, Ahne \& Kölbl 1987, Winton et al. 1987, Varner \& Lewis 1991, Neukirch et al. 1999), and using RNA-RNA blot hybridization, 7 different genogroups have been established among at least 45 aquareovirus isolates (Rangel et al. 1999). However, unlike those of mammalian and plant reoviruses in the family Reoviridae, which have been extensively studied, little information is available on the molecular characteristics of the genomes of members of the genus Aquareovirus. More recently, the sequences of the 3 largest segments of GCRV were determined (Fang et al. 2000); they are putative guanylyltransferase, viral polymerase and helicase genes. In addition, 2 sequences of other aquareoviruses have been reported. They are genome segment 10 of the Coho salmon aquareovirus (SCSV) and that of the striped bass reovirus (SBRV), which encode major outer capsid proteins (Lupiani et al. 1997a,b). Although these sequences provided some knowledge helpful for determining the diseases derived from aquatic viruses, they are still limited. In order to obtain further information on the GCRV genome and use 
available data on Aquareovirus, the full-length cDNA of GCHV genome segment S10 was cloned and the characteristics of this sequence are documented here.

Materials and methods. Virus strain, purification and RNA extraction: The CIK cell strain (Zuo et al. 1986) was used to propagate GCRV-873 (Ke et al. 1990). Virus was purified from a continuous sucrose gradient (30 to $60 \%$ sucrose in NTE [NaCl-Tris-EDTA] buffer) and centrifuged at $100000 \times g$ for $2 \mathrm{~h}$. Genomic dsRNA was extracted from the purified virus particles by $1 \%$ SDS and $10 \mu \mathrm{g} \mathrm{ml}^{-1}$ Proteinase $\mathrm{K}$ at $37^{\circ} \mathrm{C}$ for $3 \mathrm{~h}$, as well as by phenol-chloroform extraction. The purified dsRNA was treated with RQ1 RNase-free DNase (Promage) at $37^{\circ} \mathrm{C}$ for $1 \mathrm{~h}$ to remove the contamination of cell DNA, and displayed on $12.5 \%$ polyacrylamide gel (Fig. 1A).

Cloning of the $\mathbf{S 1 0}$ gene: The method described by Lambden et al. (1992) for amplification of the rotavirus dsRNA genome segments was used, with modification, to clone the GCRV S10 gene. The oligodeoxyribonucleotide primer $1\left(5^{\prime}-\mathrm{PO}_{4}\right.$-ATTTACCGCCGAGCCTGACTT- $\mathrm{NH}_{2}-3^{\prime}$ ), which was ligated to both ends of the dsRNA genome segment, was chemically synthesized and modified (Sango) to prevent self-ligation and subsequent concatenation during the RNA ligase (Life Technologies) reaction, as described by Lambden et al. (1992). The primer 1-tailed dsRNA was denatured by heating to $94^{\circ} \mathrm{C}$ for $5 \mathrm{~min}$ in $62 \%$ DMSO in the presence of primer $2\left(5^{\prime}\right.$-AAGTCAGGCTCGGCGGTAAAT-3' complementary to primer 1) (Sango) and cooled rapidly on ice. The synthesis of cDNA was carried out in reverse transcriptase reaction mix $(200 \mathrm{ng}$ dsRNA, 50 mM Tris-HCL pH 8.3, 3 mM MgCl $2,75 \mathrm{mM}$ KCL, $1 \mathrm{mM}$ DTT, $0.5 \mathrm{mM}$ each deoxynucleoside of dNTP [Promega], $40 \mathrm{U}$ RNAsin [SABC], and $200 \mathrm{U}$ reverse transcriptase [Gibco BRL]). The mixture was

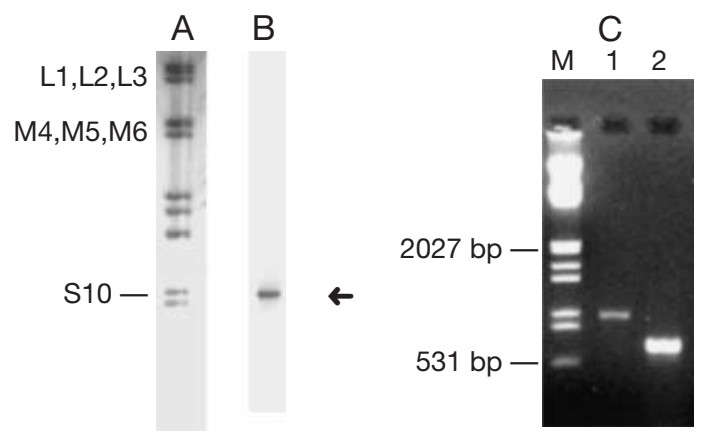

Fig. 1. Analysis of cloned S10 segment of GCRV. (A) PAGE profile of dsRNA genome of GCRV. (B) Result of Northern blotting with S10 cDNA as probe. The position of the S10 segment is indicated with $\leftarrow$. (C) Results of RT-PCR amplification of dsRNA of GCRV. M: Lambda DNA/EcoR I+ Hind III marker; Lane 1: PCR product amplified with Primer 2; Lane 2: RT-PCR product amplified with Primer $3+$ Primer 4 incubated at $37^{\circ} \mathrm{C}$ for $1 \mathrm{~h}$ and the reaction was stopped by addition of EDTA to finial $20 \mathrm{mM}$. The RNA was removed by $\mathrm{NaOH}$ and the cDNA was allowed to anneal. After extraction by phenol-chloroform and precipitation by ethanol, the annealed partial duplexes were filled in using DNA Polymerase I Large Fragment (Promage) and purified using a Glassmilk DNA purifying kit (BioStar). The amplification of cDNA was accomplished by PCR with primer 2 only using a PE9600, consisting of a denaturation step at $94^{\circ} \mathrm{C}$ for $3 \mathrm{~min}$ followed by 30 cycles of $30 \mathrm{~s}$ at $94^{\circ} \mathrm{C}, 35 \mathrm{~s}$ at $60^{\circ} \mathrm{C}$, and 2 min at $72^{\circ} \mathrm{C}$. Amplified DNA products were separated on agarose gel and the $900 \mathrm{bp}$ fragment, which corresponded in size to that calculated from the GCRV dsRNA molecular weight for S10, was excised and purified using the Glassmilk DNA purifying kit. The purified S10 was ligated directly into pGEM-T vector and transformed into DH5 $\alpha$ strain of Escherichia coli (Gibco BRL).

S10 gene sequence determination and analysis: The recombinant plasmids containing the full-length cDNA of S10 were identified according to the size of inserted segment by PCR using 2 M13 primers on PGEM-T and were purified using a plasmid DNA purification miniprep kit (Viogene). Two clones that had the full-length cDNA of S10 were used for sequencing. The nucleotide sequence of S10 was determined by Taq dye primer cycle sequencing on an ABI 310 Genetic Analyzer (Perkin Elmer). The sequence of S10 was analysed using Lasergene sequence analysis package (DNAStar).

Northern blotting and RT-PCR: Assignment of the cDNA inserts to S10 was confirmed by alkaline blotting analyses as described by Li et al. (1987). In brief, the genome dsRNA was separated in $1.0 \%$ agarose gel in Tris-acetate-EDTA buffer and transferred to a nylon memberane (Hybond) in $0.2 \mathrm{~N} \mathrm{NaOH}$ for $1 \mathrm{~h}$ at room temperature. Transferred RNA was hybridized with a cDNA probe labeled with digoxigenin-dUTP, followed by an immunodetection using Dig High Primer Labeling and Detection Starter Kit (Boehringer Mannheim). Two primers for RT-PCR, Primer 3 (5'-CCCCGATCATCACCACGAT-3') (from nucleotide 14 to 32) and Primer 4 (5'-CGCGTTCGCTGATGTAAGG-3') (from nucleotide 693 to 711 ), were synthesized according to the cDNA sequences of the cloned S10. The reverse transcription was carried out following the procedure described by Li et al. (1997).

Results and discussion. Comparing with the GCRV genome dsRNA in Fig. 1A, the clone containing the cDNA of S10 was confirmed by Northern blotting as shown in Fig. 1B, in which the cloned cDNA hybridized to genome segment S10 rather than the other genome segments. Fig. $1 \mathrm{C}$ indicates that an expected, approximately 700 bp DNA band was ampli- 
fied in the result of RT-PCR using 2 primers within the nucleotide sequence of S10. No product was found in negative control of RT-PCR with genomic DNA of CIK cell as template (data not shown).

The complete nucleotide sequence and deduced amino acid sequence of the S10 genome segment of GCRV are shown in Fig. 2. The nucleotide sequence was obtained from 2 different recombinant plasmids and constitutes a consensus sequence. The S10 gene of GCRV is 909 nucleotides long and contains a large open reading frame (ORF) preceded by a 30 bp un- translated region and followed by a $48 \mathrm{bp}$ untranslated downstream sequence. The ORF starts with an ATG codon at nucleotides 31 to 33 and ends with a TGA at nucleotide 859 to 861 . No additional ORF of significant length was detected in either the plus or minus strand RNA. Three AUG triplets, the first (ACGATGC) located at 31, the second (CACATGA) at 43 , and the third (GCTATGG) at 67, were recognized in the same reading frame and near the $5^{\prime}$ end of the mRNA strand. Since in most cases the AUG nearest the $5^{\prime}$-terminal cap is exclusively used for initiation for protein synthe-

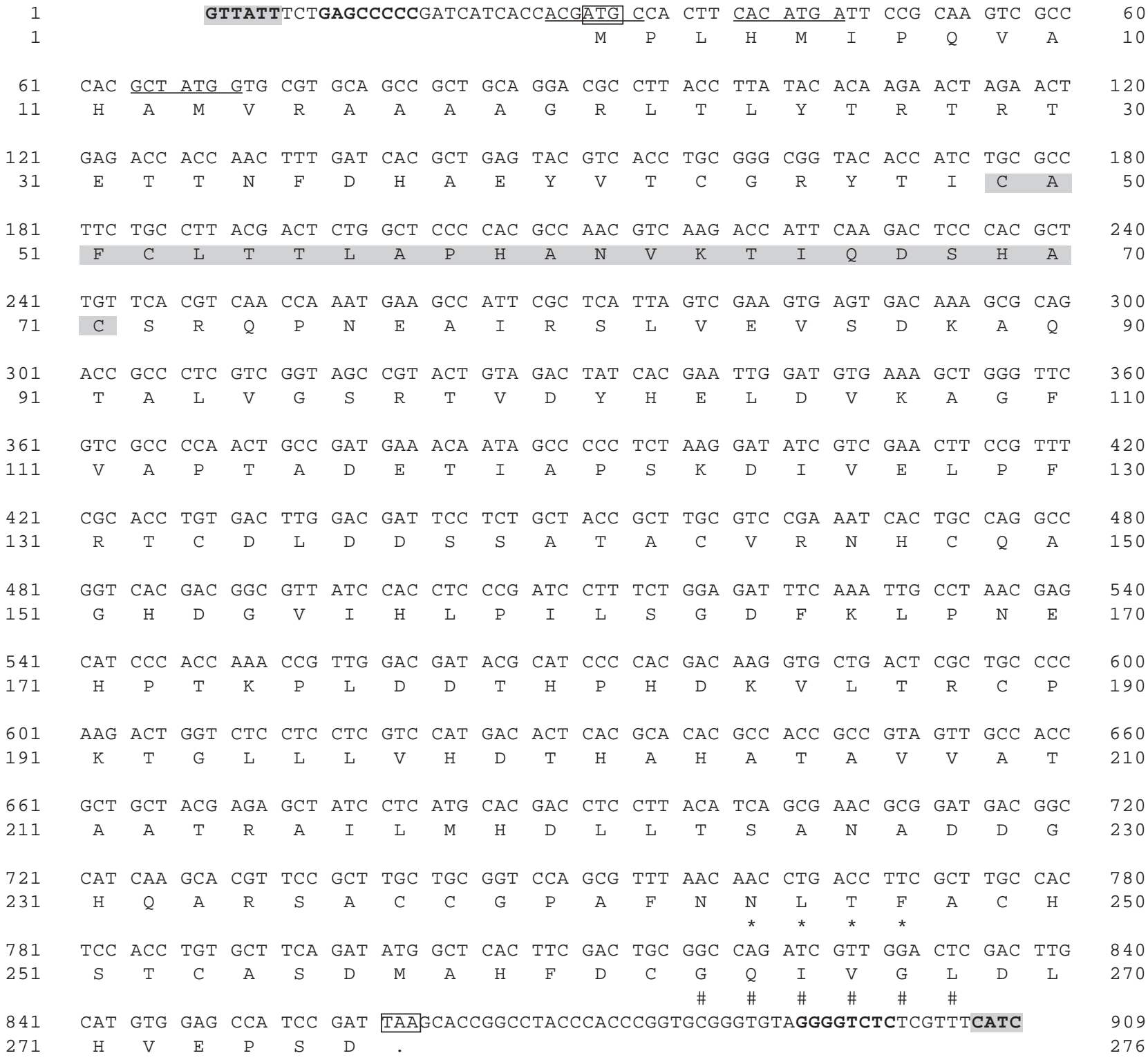

Fig. 2. Complete nucleotide sequence (presented in the cDNA form) and deduced amino acid sequence of S10 RNA segment of GCRV. The 5'- and 3'-terminal nucleotide sequences are bold and indicated with a grey background and the inverted repeats are bold. Three ATG triplets near the 5 'end of nucleotide sequence are underlined. In the amino acid sequence, the putative zincfinger motif (amino acids 49-71) is also indicated with a grey background. Possible N-glycoslation site $\left({ }^{\star}\right)$ and N-myristoylation site (\#) are indicated 
sis in a 'scanning model' (Kozak 1980), the putative methionine start codon should be the first triplet rather than the others. Interestingly, the third triplet also appeared to be potentially suitable as a functional initiator because it was consistent with the strong initiation sequence of RNNATGG ( $\mathrm{R}=$ purine nucleoside, $\mathrm{N}=$ any nucleoside) (Kozak 1981).

The GCRV S10 genome segment displays the terminal sequences 5' GUUAUU and CAUC 3', which were recognized to be conservative in the GCRV RNA segments (authors' unpubl. data). Moreover, the 3 '-terminal sequence CAUC 3' was found to be the same as that of segment S10 of SBRV (Lupiani et al. 1997b), another member of Aquareovirus. In addition, a putative inverted repeat sequence was identified adjacent to the terminal sequence. The $5^{\prime}$ end sequence, GAGCCCCC at 10 to 17, and its 3' end inverted repeat sequence, GGGGTCTC at 892 to 899, are almost completely complementary.

The conserved terminal sequences are broadly reported in members of the family Reoviridae. They may be important in sorting and packing functions of the virus (Anzola et al. 1987). Analysis of the terminal nucleotide sequences is of interest in relation to understanding mechanisms of transcription and replication. The same 3' terminal sequence between GCRV S10 and SBRV S10 implied relatively closer relations between them. In addition, like other reoviruses, a domain of inverted repeat adjacent to the 5' and 3' terminus always plays an important role in distinguishing this genome segment from other segments (Anzola et al. 1987).

The ORF is considered to encode a protein of 276 amino acids with a deduced molecular weight of approximately $29.7 \mathrm{kDa}$. The possible modification sites, an N-glycosylation site present at 244 to 247 and an $\mathrm{N}$-myristoylation site at 263 to 268, were indicated and near the $\mathrm{C}$ terminus of the polypeptide (Fig. 2). Using the BLAST programs (Altschul et al. 1997), we were unable to find substantial similarity with either the nucleotide or the amino acid sequence of S10 and sequences of characterized animal reovirus and plantinfecting reovirus. However, some similarities in GCRV S10, SCSV S10 and SBRV S10 were observed. They not only have similar segment lengths, but the molecular weights of their deduced peptides are also analogous. Moreover, they are all segment 10 of the viruses in the same genus, i.e. Aquareovirus. We compared the deduced amino acid sequence of GCRV S10 with those of SCSV S10 and SBRV S10 using the Lasergene program (DNAStar). The predicted protein encoded by GCRV S10 showed a similarity index of 17.1 (Lipman \& Pearson 1985) with SCSV S10 in a 173 amino acid overlap, and of 30 with SBRV S10 in only a 20 amino acid overlap.
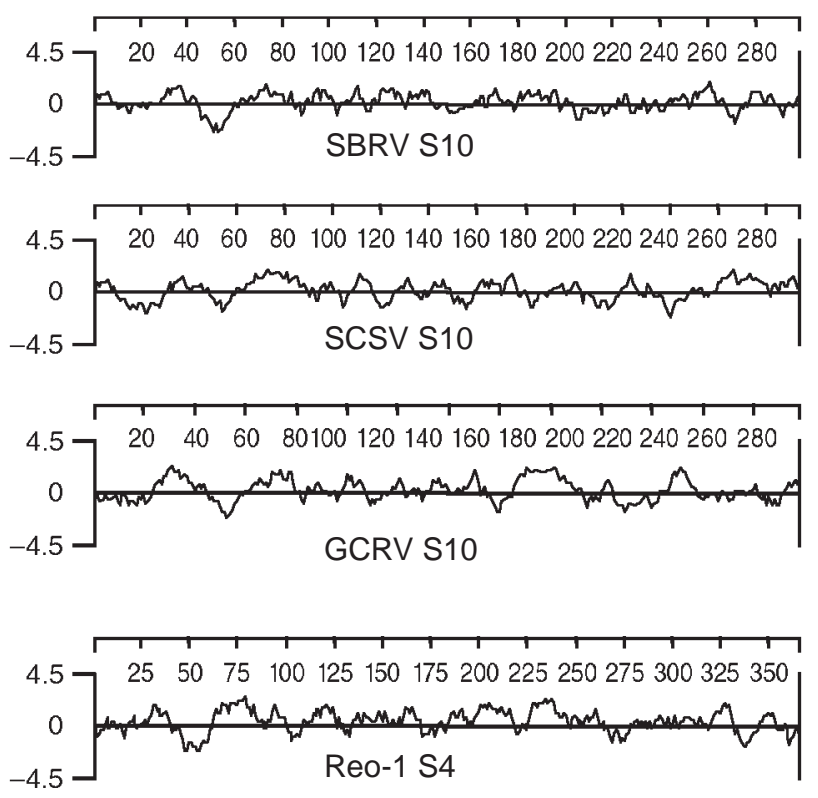

Fig. 3. Hydrophilic plots of deduced proteins encoded by SBRV S10, SCSV S10, GCRV S10 and Reo-1 S4

Although it can be proposed that GCRV S10 may correspond to SCSV S10 to a low extent and may not be related to SBRV S10 with respect to amino acid sequences, the hydrophilic plots (Fig. 3) produced according to the method of Kyte \& Doolittle (1982) significantly showed that the predicted proteins encoded by GCRV S10, SCSV S10 and SBRV S10 had very similar profiles, particularly in the regions of the N-terminal half. Thus, it can be predicted that their amino acid sequences may have an analogous molecular conformation. Furthermore, it is very likely that this analogous conformation leads to functional similarities among the deduced proteins of the S10 segments of these aquareoviruses.

The deduced amino acid sequence of GCRV S10 possesses a $\mathrm{CX}_{2} \mathrm{CX}_{16} \mathrm{HX}_{1} \mathrm{C}$ sequence within the $\mathrm{N}$ terminus, from residues 49 to 71 , which is analogous to the zinc-finger domain ( $\mathrm{CCHC}$ ) identified within the mammalian reovirus (MRV) $\sigma 3$ protein encoded by genome S4 gene (Mabrouk \& Lemay 1994). Comparable in position, the zinc-finger sequence $\mathrm{CX}_{2} \mathrm{CX}_{15} \mathrm{HHX}_{1} \mathrm{C}$ is from amino acids 51 to 73 within the MRV $\sigma 3$ protein. Notably, we also recognized the zinc-finger domains within deduced amino acid sequences of SCSV $\mathrm{S} 10\left(\mathrm{CX}_{2} \mathrm{CX}_{16} \mathrm{HX}_{1} \mathrm{C}\right)$ and SBRV S10 $\left(\mathrm{CX}_{2} \mathrm{CX}_{15} \mathrm{HX}_{1} \mathrm{C}\right)$ (Fig. 4), although they had not been indicated in the original reports (Lupiani et al. 1997a,b). Moreover, they are located at the same or a position very similar to that of GCRV S10, namely, at residues from 50 to 71 and from 49 to 71 , respectively. Thus, the $\mathrm{CX}_{2} \mathrm{CX}_{15}$ ${ }_{16} \mathrm{HX}_{1} \mathrm{C}$ was found to be the special zinc-finger 


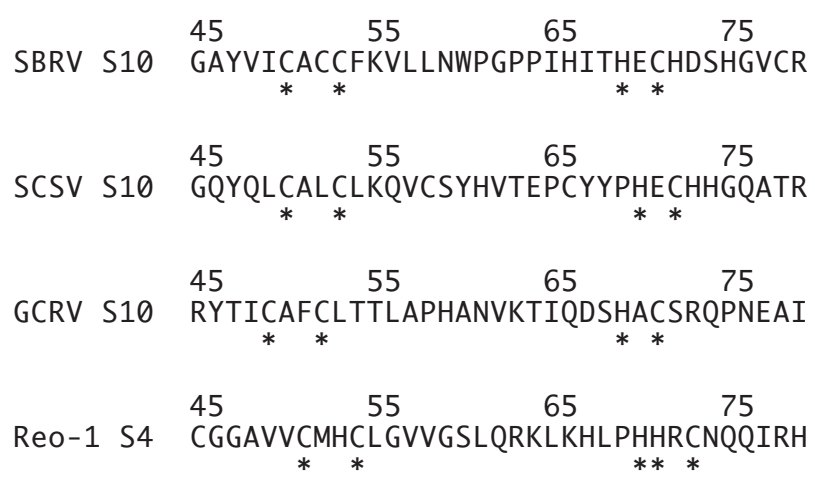

Fig. 4. Sequences of the putative zinc-binding sites in deduced amino acid sequences of GCRV S10, SBRV S10, SCSV S10, and Reo-1 S4. *Residues of the putative zinc fingers. Numbers refer to the amino acid positions

sequence shared by the deduced peptide of the S10 segments of these aquareoviruses and MRV $\sigma 3$. Since the most probable functional motif was identified as being a form $\mathrm{CX}_{2} \mathrm{CX}_{15} \mathrm{HX}_{2} \mathrm{C}$ (Mabrouk \& Lemay 1994), the function of the zinc-finger motifs indicated here requires further study. In previous reports, the zinc-finger domains may be required by some proteins to maintain proper conformation (McIntyre et al. 1993) and may also be involved in protein-protein interactions (Cunningham et al. 1991).

Since the comparable zinc-finger domain exists in the MRV $\sigma 3$ protein, the hydrophilic plot of the reovirus serotype-1 (Reo-1) S4 (Seliger et al. 1992), as an ex-ample of MRV, was given together with those of aquareoviruses. Surprisingly, many profiles were also similar to those of aquareoviruses, although MRV belongs to another genus, Orthoreovirus. In particular, the N-terminal 160 amino acids of Reo-1 S4 are very like those of GCRV S10, and the C-terminal is analogous to those of GCRV S10 and SBRV S10, suggesting that the deduced amino acid sequences of the aquareovirus S10 may share some functional similarity with MRV $\sigma 3$ protein. In addition, it was notable that the terminal sequences of MRV S4, 5' GCUAUU and CAUC 3' are very similar to that of GCRV S10. From an evolutionary standpoint, it is likely that the GCRV S10 and the MRV S4 have evolved from a common ancestral precursor.

Since SCSV S10, SBRV S10, and Reo-1 S4 were previously revealed to encode major outer capsid proteins (Atwater et al. 1986, Lupiani et al. 1997a,b), and since similarities have been shown between the zincfinger sequences and hydrophilic plots of GCRV S10 and the above viral segments, it can be predicted that the deduced peptide of GCRV S10 is an outer capsid protein. In addition, the smallest segment of the GCRV genome, S11, has already been assigned to encode nonstructural proteins (authors' unpubl. data), suggesting that the deduced peptide encoded by GCRV S10 may be the smallest outer capsid protein.

We compared the molecular weight of the deduced peptide of GCRV S10 with that of the proteins isolated from GCRV virions as described by Ke et al. (1992). In that report, the smallest outer capsid protein of GCRV is $27 \mathrm{kDa}$, slightly smaller than the $29.7 \mathrm{kDa}$ protein deduced. Considering previous studies on aquareoviruses (Winton et al. 1987, Hsu YL et al. 1989, Subramanian et al. 1994) which reported that the smallest structural proteins all were approximately 31 to $36 \mathrm{kDa}$, it is possible that a deviation from reading data on the SDS-PAGE existed in the Ke et al. report. Moreover, the same protein identified in another report (Wang et al. 1990) was $31 \mathrm{kDa}$, which approximates the size of deduced product of S10. Our study will serve as a preliminary for future investigations. In order to learn more about the structure and function of the protein encoded by GCRV S10 and its similarities with other proteins, additional work including biochemical assays and immunological analysis should be performed.

Acknowledgements. This work was supported by Grant No. ZYJ01-04 from the China-Israel fund for scientific and strategic research and development, and by Grant No. 990205FB05 from FEBL, the state key laboratory of Freshwater Ecology and Biotechnology.

\section{LITERATURE CITED}

Ahne W, Kolbl O (1987) Occurrence of reovirus in European cyprinid fishes (Tinca tinca Lin., Leucisus cephalus Lin.) J Appl Ichthyol 3:139-141

Altschul SF, Madden TL, Schiffer AA, Zhang J, Zhang Z, Miller W, Lipman DJ (1997) Gapped BLAST and PSIBLAST: a new generation of protein database search programs. Nucleic Acids Res 25:3389-3402

Anzola JV, Xu Z, Asamizu T, Nuss DL (1987) Segment specific inverted repeats found adjacent to conserved terminal sequences in wound tumor virus genome and defective interfering RNAs. Proc Natl Acad Sci USA 84:8301-8305

Atwater JA, Munemitsu SM, Samuel CE (1986) Biosynthesis of reovirus-specified polypeptides. Molecular complementary DNA cloning and nucleotide sequence of the reovirus serotype 1 Lang strain S4 messenger RNA which encodes the major capsid surface polypeptide $\sigma 3$. Biochem Biophys Res Commun 136:183-192

Chen Y, Jiang Y (1983) Studies on the morphological and physico-chemical characterization of the hemorrhagic virus of grass carp. KeXue TongBao (Foreign Lang Edn) 28:1138-1140

Cunningham BC, Mulkerrin MG, Wells JA (1991) Dimerization of human growth hormone by zinc. Science 253: $545-548$

Ding Q, Yu L, Wang X, Ke L (1991) Study on infecting other fishes with grass carp hemorrhagic virus. Chin J Virol 6(4):371-373 (in Chinese with English abstract)

Fang Q, Attoui H, Biagini JF, Zhu Z, de Micco P, de Lamballerie X (2000) Sequence of genome segments 1, 2, and 3 of 
the grass carp reovirus (Genus aquareovirus, family reoviridae). Biochem Biophys Res Commun 274(3):762-766

Hedrick RP, Rosemark R, Aronstein D, Winton JR, McDowell T, Amend DF (1984) Characteristics of a new reovirus from channel catfish (Ictalurus punctatus). J Gen Virol 65: 1527-1534

Hsu Y, Chen B, Wu J (1989) Characteristics of a new reo-like virus isolated from landlocked salmon (Oncorhynchus Masou Brevoort). Fish Pathol 24(1):37-45

Ke L, Fang Q, Cai Y (1990) Characteristics of a new isolation of hemorragic virus of grass carp. Acta Hydrobiol Sin 14(2):153-159 (in Chinese with English abstract)

Ke L, Wang W, Fang Q, Cai Y (1992) Studies on the in vitro translation of grass carp hemorrhage virus and its proteins. Chin J Virol 8(2):169-173 (in Chinese with English abstract)

Kozak M (1980) Evaluation of the 'scanning model' for initiation of protein synthesis in eukaryotes. Cell 22:459-467

Kozak M (1981) Possible role of flanking nucleosides in recognition of the AUG initiator codon by eukaryotic ribosomes. Nucleic Acids Res 6:5233-5250

Kyte J, Doolittle RF (1982) A simple method for displaying the hydropathic character of a protein. J Mol Biol 157:105-132

Lambden PR, Cooke SJ, Caul EO, Clarke IN (1992) Cloning of noncultivatable human rotavirus by single primer amplification. J Virol 66:1817-1822

Li JK, Parker B, Kowlik T (1987) Rapid alkaline blottransfer of viral dsRNAs. Anal Biochem 163:210-218

Li J, Wang T, Yi Y, Liu H, Lu R, Chen H (1997) A detection method for grass carp hemorrhagic virus (GCHV) based on a reverse transcription-polymerase chain reaction. Dis Aquat Org 29:7-12

Li J, Wang T, Lu R, Chen H (1999) Advance in research of hemorrhagic virus of grass carp. Oceanol Limnol Sin 30(4): 445-453 (in Chinese with English abstract)

Lipman DJ, Pearson WR (1985) Rapid and sensitive protein similarity searches. Science 227:1435-1441

Lupiani B, Reddy SM, Samal SK (1997a) Sequence analysis of genome segment 10 encoding the major outer capsid protein (VP7) of genogroup B aquareovirus and its relationship with the VP7 protein of genogroup A aquareovirus. Arch Virol 142(12):2547-2552

Lupiani B, Reddy SM, Subramanian K, Samal SK (1997b) Cloning, sequence analysis and expression of the major outer capsid protein gene of an aquareovirus. J Gen Virol 78(6):1379-1383

Editorial responsibility: Jo-Ann Leong,

Corvallis, Oregon, USA
Mabrouk T, Lemay G (1994) Mutations in a CCHC zinc-binding motif the reovirus $\sigma 3$ protein decrease its intracellular stability. J Virol 68:5287-5290

McIntyre MC, Frattini MG, Grossman SR, Laimins LA (1993) Human papillomavirus type 18 E7 protein requires intact Cys-X-X-Cys motif for zinc binding, dimerization, and transformation but not for $\mathrm{Rb}$ binding. J Virology 67: 3142-3150

Neukirch M, Haas L, Lehmann H, Messeling VV (1999) Preliminary characterization of a reovirus isolated from golden ide Leuciscus idus melanotus. Dis Aquat Org 35: 159-164

Plumb JA, Bowser PR, Grizzle JM, Mitchell AJ (1979) Fish viruses: a double-stranded RNA icosahedral virus from a North American cyrinid. J Fish Res Board Can 36: 1390-1394

Rangel AAC, Rockemann DD, Hetrick FM, Samal SK (1999) Identification of grass carp haemorrhage virus as a new genogroup of aquareovirus. J Gen Virol 80:2399-2402

Seliger LS, Giantini M, Shatkin AJ (1992) Translation effects and sequence comparisons of the three serotypes of the reovirus S4 gene. Virology 187:202-210

Subramanian K, McPhillips TH, Samal SK (1994) Characterization of the polypeptides and determination of genome coding assignments of an aquareovirus. Virology 205: 75-81

Varner PW, Lewis DH (1991) Characterazation of a virus associated with head and lateral line erosion syndrome in marine angel fish. J Aquat Anim Health 3:198-205

Wang T, Chen H, Liu H, Yi Y, Guo W (1994) Preliminary studies on the susceptibility of Gobiocypris rarus to hemorrhagic virus of grass carp. Acta Hydrobiol Sin 18(2): 144-149 (in Chinese with English abstract)

Wang W, Chen Y, Ke L, Cai Y (1990) Fine structure, genome and polypeptides of a grass carp hemorrhage virus (GCHV) isolate from the South Lake in Wuhan Hemorrhage. Chin J Virol 6(1):46-49 (in Chinese with English abstract)

Winton JR, Lannan CN, Fryer JL, Hedrick RP, Meyers TR, Plumb JA, Yamamoto T (1987) Morphological and biochemical properties of four members of a novel group of reovirus isolated from aquatic animals. J Gen Virol 68: 353-364

Zuo W, Qian H, Xu Y, Du S, Yang X (1986) A cell line derived from the kidney of grass carp. J Fish China 10(1):11-17 (in Chinese with English abstract)

Submitted: April 29, 2000; Accepted: October 25, 2000

Proofs received from author(s): January 10, 2001 